\title{
Modelado Matemático de la Posición del Centro de Masa de un Robot de Tracción Diferencial. Un Enfoque desde la Mecánica Lagrangiana
}

\author{
Juan P. Cardona ${ }^{(1) \star}$, John J. Leal ${ }^{(2)}$ y José L. Ramirez ${ }^{(3)}$
}

(1) Univ. Cooperativa de Colombia, Facultad de Ingeniería, Av. Caracas No. 37-15, Bogotá D.C. Colombia Colombia. (e-mail: juan.cardonag@campusucc.edu.co)

(2) Univ. Nac. de Colombia, Facultad de Ing. y Administración, Km. 32 № 12-00, Palmira, Colombia

Colombia. (e-mail: jlealgom@unal.edu.co)

(3) Univ. Militar Nueva Granada, Facultad de Estudios a Distancia, Km 2 de la vía Cajica-Zipaquira, Cajica, Colombia. (e-mail: jose.ramirez@unimilitar.edu.co)

${ }^{*}$ Autor a quien debe ser enviada la correspondencia

Recibido Mar. 1, 2018; Aceptado May. 15, 2018; Versión final Jul. 21, 2018, Publicado Dic. 2018

\section{Resumen}

El objetivo del artículo es desarrollar y validar un modelo matemático para determinar la posición y orientación del centro de masa de un robot móvil (Kit LEGO NXT 2.0) en función de las tensiones aplicadas a los servomotores de las ruedas, utilizando modelos cinemáticos y dinámicos del robot con enfoque desde la mecánica Lagrangiana. Los resultados obtenidos en el experimento fueron procesados utilizando el software Tracker, y se compararon estadísticamente mediante una prueba t-student con los resultados obtenidos al realizar la simulación del modelo en el Simulink de Matlab. Al comparar los valores se obtuvieron resultados satisfactorios, razón por la cual se puede concluir que el modelo establecido da cuenta adecuada del comportamiento del centro de masa del robot.

\section{Mathematical Modeling of Mass Center Position of Differential Traction Robot. An Approach from Lagrangian Mechanics}

\begin{abstract}
The target of this paper is to develop and validate a mathematical model to determine the center of mass' position and orientation of a mobile robot (NXT LEGO Kit 2.0). The model takes into account input voltage applied to wheel's servomotors and is formulated using dynamic and kinematics of the robot with a Lagrangian mechanics approach. Experimental results are obtained using the so-called Tracker software. Subsequently, data is processed using a t-student test, comparing experimental data with simulation results, which are obtained executing the model in Matlab-Simulink. As a result, the mathematical model accurately describes the behavior of the robot's center of mass.
\end{abstract}

Keywords: differential mobile robot; mathematical modeling; center of mass; dynamics; Lagrangian mechanics 


\section{INTRODUCCIÓN}

Un robot móvil se define como un sistema electromecánico capaz de desplazarse en forma autónoma de un punto a otro en un determinado espacio de trabajo. De acuerdo al tipo de locomoción que emplean los robots móviles para desplazarse se clasifican en tres categorías, robots de ruedas (Alexander y Madoocks, 1989; Campion, et al. 1996; Muir y Neuman, 1987), robots de patas (Tood, 1985) y robots orugas (Granosik y Borestein, 2005). Un robot móvil diferencial consta de dos ruedas activas conectadas directamente o mediante un tren de engranajes a dos servomotores, los cuales se mueven cuando aplicamos tensión a su entrada. Cuando las tensiones son aplicadas se inducen velocidades angulares sobre las ruedas, lo que produce cambios en la dirección del robot, de tal forma que si las tensiones son iguales el robot se desplazará en línea recta, en tanto que cuando una de las tensiones sea mayor que la otra, el robot describe una trayectoria curva.

Las aplicaciones de los robots móviles son innumerables (Ortigoza, et al. 2012): "la exploración planetaria, minera, marítima, reconocimiento de terreno, inspección y vigilancia, misiones de búsqueda y rescate de personas, limpieza de desechos peligrosos y en ambientes diversos, asistencia médica, entretenimiento, etc", además de utilizarse en la industria metalmecánica, investigación militar, industria química, transporte y agricultura entre otros. Se han estudiado modelos cinemáticos de robots móviles diferenciales (Gomez y Gomez, 2017; Muir, 1987), algunos de los cuales se utilizan para diseñar controladores con su respectiva validación en forma experimental (Chung, 2001), también se han diseñado modelos dinámicos para robots móviles diferenciales con tres ruedas desde la perspectiva de la mecánica Lagrangiana, con verificación experimental (Buratowski y Giergiel, 2010). En la literatura varios artículos han estudiado robots móviles diferenciales desde la perspectiva cinemática y dinámica principalmente enfocados para el diseño de controladores y utilizando la mecánica Lagrangiana para la formulación de los modelos (Campion, et al., 1996; Fierro y Lewis, 1995; Klančar, et al., 2007; Mahler y Haase, 2013; Muir y Neuman, 1987; Esmaeili, et al., 2017; Salem, 2013).

El sistema robótico que se conoce como LEGO NXT 2.0 se usa con fines académicos y de investigación (Gomez-de-Gabriel, et al. 2011), se compone básicamente por piezas plásticas (Uniones, ruedas, pasadores, servomotores) y un ladrillo inteligente que debido a su gran versatilidad permite diferentes ensamblajes. El robot de tracción diferencial cuenta con dos servomotores, dos ruedas dos cables de comunicación, una rueda loca, dos pasadores y cuatro piezas de unión. El ensamblaje propuesto para el estudio realizado se muestra en la figura 1.

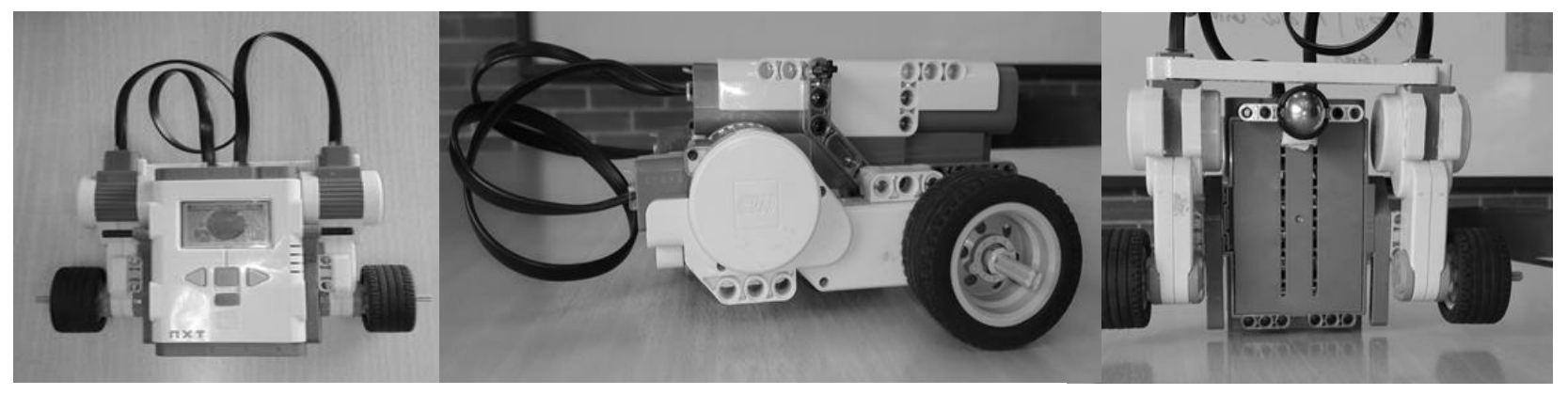

Fig. 1: Ensamblaje del robot móvil Kit LEGO NXT.

En éste artículo se desarrolla y valida un modelo matemático que considera las características cinemáticas y dinámicas del robot LEGO NXT 2.0, aplicando los principios energéticos de la mecánica Lagrangiana para determinar la posición del centro de masa del robot en función de las tensiones aplicadas a los servomotores de las ruedas, teniendo como entradas las tensiones aplicadas a la armadura de cada servo y como salida la trayectoria en el plano XY ideal sin obstáculos. El modelo se simuló utilizando el Software SIMULINK de Matlab y se validó contrastando los resultados experimentales con datos obtenidos utilizando el software TRACKER, paquete de análisis de video que permite seguir objetos que estén resaltados, determinar su posición espacial, o en función del tiempo, realizar gráficas y análisis entre otras. En éste caso el software es adecuado puesto que el robot no cuenta con sensores que suministren información de la posición del robot en el plano $\mathrm{XY}$. Los datos experimentales y los resultados de la simulación fueron comparados estadísticamente mediante una prueba t-student.

\section{CONSIDERACIONES GENERALES DEL MODELO MATEMÁTICO}

El diseño del modelo matemático tiene como variables de entrada las tensiones aplicadas a los servomotores y las variables de salida corresponden a los valores de posición del centro de masa del robot representada en coordenadas $(\mathrm{x}, \mathrm{y})$. Dadas las tensiones de entrada y utilizando el modelo electromecánico de los 
servomotores se pueden obtener sus corrientes y velocidades angulares, las cuales resultan no ser apropiadas para aplicarlas directamente a las ruedas del robot, siendo necesaria la reducción de su magnitud por medio de un tren de engranajes rectos. La formulación del modelo dinámico del sistema completo (teniendo en cuenta el cuerpo del robot y ruedas, la masa completa del robot, la masa de las ruedas, los momentos de inercia del robot y de las ruedas), cuyas variables de entrada corresponden a las velocidades angulares de las ruedas, y las variables de salida los momentos torsores aplicados y las aceleraciones angulares de las ruedas. El modelo se planteó utilizando el enfoque energético de la Mecánica Lagrangiana.

Integrando las aceleraciones angulares con respecto al tiempo, se obtienen las velocidades angulares que corresponden a las entradas del modelo cinemático, a partir del cual se obtienen las posiciones y orientación $(\mathrm{x}, \mathrm{y})$ del centro de masa del robot. Para la formulación del modelo se tienen en cuenta las siguientes consideraciones: 1 . En el robot LEGO NXT 2.0, tiene partes flexibles, que para el análisis serán tratadas como cuerpos rígidos; 2 . La fricción en el punto de contacto entre la rueda y el punto de contacto sobre la superficie de rodadura, es lo suficientemente grande para que no ocurra deslizamiento (rodadura pura); 3 . El eje de la rueda permanece perpendicular a la superficie en todo momento; y 4 . El robot se desplaza sobre una superficie plana.

\section{MODELO CINEMÁTICO DEL ROBOT}

Para el modelo cinemático del robot se tiene en cuenta su geometría referida a un marco inercial fijo (X,Y) como se muestra en la figura $2 a$, la velocidad del centro de masa del robot $V(t)$ se escribe en función de tres aspectos: i) Las velocidades tangenciales de las ruedas derecha e izquierda ( $\mathrm{V}_{1}$ y $\left.\mathrm{V}_{2}\right)$, (ii) la velocidad angular de las ruedas $\dot{\theta}$ desde el punto $\mathrm{Po}_{\mathrm{O}}=(\mathrm{x}, \mathrm{y})$ iii) y el ángulo de avance $\psi$ (curso del robot o guiñada) en el marco de referencia global.

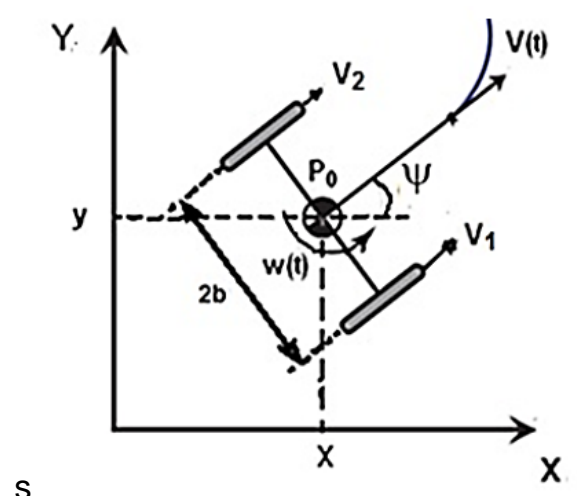

(a)

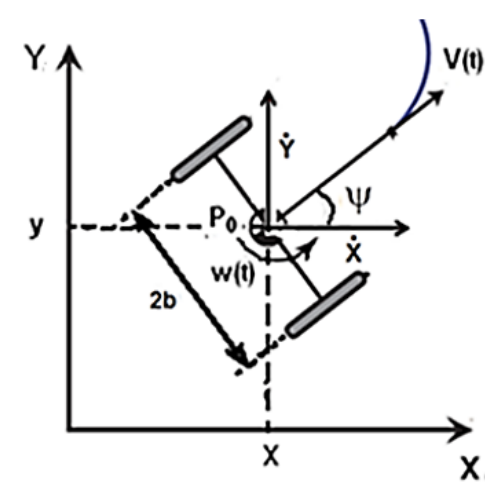

(b)

Fig. 2: Variables cinemáticas del robot diferencial.

Las velocidades tangenciales del robot $V_{1}$ y $V_{2}$ del modelo cinemático para la posición del centro de masa se escriben en las ecuaciones (1).

$\mathrm{V}_{1}=\dot{\theta}_{1} \mathrm{r}, \mathrm{V}_{2}=\dot{\theta}_{2} \mathrm{r}$

El ángulo de giro del centro de masa del robot LEGO $\psi$ se obtiene con base en las relaciones geométricas entre el movimiento de cada una de las ruedas, como se muestra en la figura 3, en la cual se observa que él ángulo de giro del centro de masa del robot es igual al ángulo del arco sostenido por las trayectorias $S_{1} \circ S_{2}$.

La rueda izquierda sostiene un arco de radio $\mathrm{X}$ por lo que la distancia recorrida por ésta rueda está dada por la ecuación 2:

$\mathrm{S}_{2}=\mathrm{X} \Delta \psi=\mathrm{r} \Delta \theta_{2}$

La rueda derecha recorre una distancia que está dada por la ecuación 3:

$\mathrm{S}_{1}=(\mathrm{X}+2 \mathrm{~b}) \Delta \psi=\mathrm{r} \Delta \theta_{1}$

Al calcular la diferencia entre $S_{1}-S_{2}$ se obtiene la ecuación (4):

$S_{1}-S_{2}=2 b \Delta \psi=r \Delta \theta_{1}-r \Delta \theta_{2}$ 
Al despejar resulta la ecuación (5):

$\Delta \psi=\frac{\mathrm{r} \Delta \theta_{1}-\mathrm{r} \Delta \theta_{2}}{2 \mathrm{~b}}$

Dividiendo entre $\Delta t$ en la ecuación 5 se obtiene la ecuación 6 :

$\frac{\Delta \psi}{\Delta \mathrm{t}}=\frac{\mathrm{r} \Delta \theta_{1}-\mathrm{r} \Delta \theta_{2}}{2 \mathrm{~b} \Delta \mathrm{t}}$

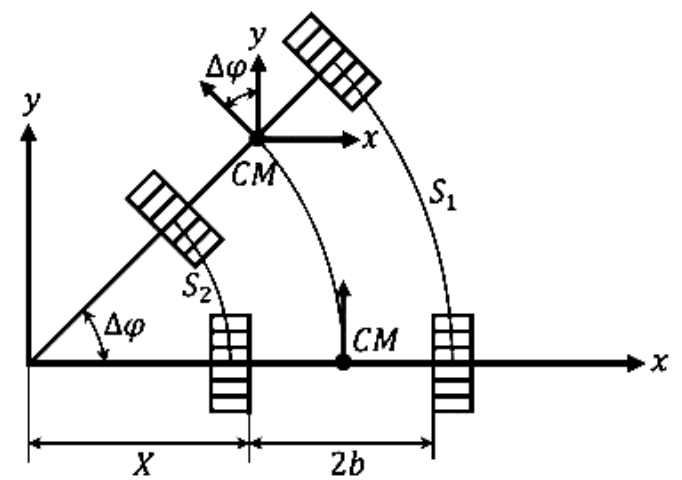

Fig. 3: Cambio de posición angular del centro de masa del robot.

Tomando el límite cuando $\Delta \mathrm{t} \rightarrow 0$, la velocidad angular del centro de masa del robot $\dot{\psi}$ es:

$\dot{\psi}=\lim _{\Delta \mathrm{t} \rightarrow 0} \frac{\Delta \psi}{\Delta \mathrm{t}}=\lim _{\Delta \mathrm{t} \rightarrow 0} \frac{\mathrm{r} \Delta \theta_{1}-\mathrm{r} \Delta \theta_{2}}{2 \mathrm{~b} \Delta \mathrm{t}}=\frac{\mathrm{r}\left(\dot{\theta}_{1}-\dot{\theta}_{2}\right)}{2 \mathrm{~b}}$

Considerando el robot como un cuerpo rígido, la velocidad lineal de su centro de masa $\mathrm{V}_{\mathrm{CM}}$ se obtiene considerando las expresiones vectoriales de velocidades relativas para cada una de las ruedas como se aprecia en la figura 4.

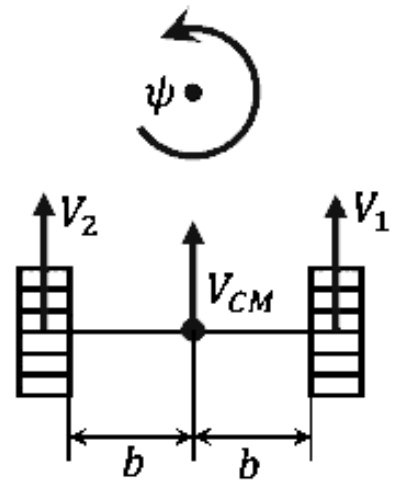

Fig. 4: Representación de las velocidades de las ruedas del robot y de su centro de masa

Las velocidades de las ruedas 1 y 2 respecto al centro de masa del robot se muestran en las ecuaciones 8 :

$\overrightarrow{\mathrm{V}}_{1}=\overrightarrow{\mathrm{V}_{\mathrm{CM}}}+\overrightarrow{\mathrm{V}_{1 / \mathrm{CM}}}, \quad \overrightarrow{\mathrm{V}}_{2}=\overrightarrow{\mathrm{V}_{\mathrm{CM}}}-\overrightarrow{\mathrm{V}_{2 / \mathrm{CM}}}$

Las velocidades relativas de las ruedas con respecto al centro de masa están definidas en las ecuaciones 9 :

$\mathrm{V}_{1 / \mathrm{CM}}=\mathrm{V}_{2 / \mathrm{CM}}=\dot{\psi} \mathrm{b}$

Reemplazando las ecuaciones 9 en las ecuaciones 8 , se obtienen la velocidad lineal 10:

$\mathrm{V}_{1}=\mathrm{V}_{\mathrm{CM}}+\dot{\psi} \mathrm{b}, \mathrm{V}_{2}=\mathrm{V}_{\mathrm{CM}}-\dot{\psi} \mathrm{b}$

Reescribiendo las ecuaciones e igualando las expresiones de $\dot{\psi}$ b se obtiene la ecuación 11:

$\mathrm{V}_{1}-\mathrm{V}_{\mathrm{CM}}=\mathrm{V}_{\mathrm{CM}}-\mathrm{V}_{2}$

Despejando la velocidad del centro de masa $\mathrm{V}_{\mathrm{CM}}$, resultan las ecuaciones 12 : 
$\mathrm{V}_{\mathrm{CM}}=\frac{\mathrm{V}_{1}+\mathrm{V}_{2}}{2}=\frac{\mathrm{r}\left(\dot{\theta}_{1}+\dot{\theta}_{2}\right)}{2}$

La velocidad angular del centro de masa $\dot{\psi}$ se escribe en las ecuaciones 13:

$\dot{\psi}=\frac{\mathrm{V}_{1}+\mathrm{V}_{2}}{2 \mathrm{~b}}=\frac{\mathrm{r}\left(\dot{\theta}_{1}-\dot{\theta}_{2}\right)}{2 \mathrm{~b}}$

Para obtener las coordenadas de la posición del centro de masa del robot, es necesario descomponer la velocidad lineal del centro de masa del robot en sus componentes rectangulares $(\dot{X}, \dot{Y})$ en el plano XY como se muestra en la figura $2 \mathrm{~b}$ de esta manera las expresiones se escriben en la ecuación 14.

$$
\dot{\mathrm{X}}=\mathrm{V}_{\mathrm{CM}} \operatorname{Cos} \psi, \dot{\mathrm{Y}}=\mathrm{V}_{\mathrm{CM}} \operatorname{Sen} \psi
$$

Reemplazando los valores correspondientes de VCM y $\dot{\psi}$ de las ecuaciones 12 y 13 en la ecuación 14 y escribiendo el resultado en forma vectorial se obtiene:

$$
\left[\begin{array}{lll}
\dot{X}, & \dot{Y} & \dot{\psi}
\end{array}\right]=\left[\left(\frac{\dot{\theta}_{1}+\dot{\theta}_{2}}{2}\right) r \operatorname{Cos} \psi,\left(\frac{\left.\dot{\theta}_{1}+\dot{\theta}_{2}\right)}{2}\right) r \operatorname{Sen} \psi, \frac{\left(\dot{\theta}_{1}-\dot{\theta}_{2}\right) r}{2 b}\right]
$$

Para obtener la posición y orientación del centro de masa del robot a partir de las velocidades angulares de cada una de las llantas y con una posición inicial se integran las expresiones de la ecuación 15 obteniendo 16:

$$
[\mathrm{X}(\mathrm{t}), \quad \mathrm{Y}(\mathrm{t}), \quad \psi(\mathrm{t})]=\left[\begin{array}{lll}
\mathrm{X}_{0}, & \mathrm{Y}_{0}, & \psi_{0}
\end{array}\right]+\left[\int_{0}^{\mathrm{t}}\left(\frac{\dot{\theta}_{1}+\dot{\theta}_{2}}{2}\right) \mathrm{r} \operatorname{Cos}(\psi(\mathrm{t})) \mathrm{dt}, \int_{0}^{\mathrm{t}}\left(\frac{\dot{\theta}_{1}+\dot{\theta}_{2}}{2}\right) \mathrm{rSen} \psi((\mathrm{t})) \mathrm{dt}, \quad \int_{0}^{\mathrm{t}} \frac{\left(\dot{\theta}_{1}-\dot{\theta}_{2}\right) \mathrm{rdt}}{2 \mathrm{~b}}\right]
$$

\section{MODELO DINÁMICO DEL ROBOT}

Para el cálculo del modelo dinámico de un robot es necesario inicialmente determinar los parámetros inerciales de base que constituyen el conjunto mínimo de valores de inercias y masas a tener en cuenta (Salinas, 2009). Tales parámetros permiten eliminar aquellos que no tienen efecto sobre el modelo, agrupando otros con el fin de simplificar las ecuaciones finales del modelo dinámico.

El modelo dinámico del robot relaciona las masas e inercias del cuerpo del robot y la aceleración angular en cada rueda $\ddot{\theta}$ en función del momento de torsión aplicado a cada motor $\tau$. Al Considerar el cuerpo del robot y sus ruedas como cuerpos rígidos de masas $\mathrm{M}$ y $\mathrm{m}$ respectivamente, las ecuaciones dinámicas se determinan utilizando los principios energéticos expuestos en la mecánica Lagrangiana, donde $(\ell)$ es el Lagrangiano del robot se calcula mediante la suma del Lagrangiano de cada una de las ruedas $\left(\ell_{1}, \ell_{2}\right)$ más el Lagrangiano del bloque del robot $\left(\ell_{3}\right)$ tal como se muestra en la ecuación 17.

$$
\ell=\ell_{1}+\ell_{2}+\ell_{3}
$$

La energía potencial del robot permanece constante debido a que la altura del centro de masa del robot en el sistema $X Y$ es la misma en todo momento, reduciéndose el cálculo del Lagrangiano a la suma de la energía cinética del bloque del robot $\left(\mathrm{K}_{\mathrm{c}}\right)$ más la energía cinética de cada rueda $\left(\mathrm{K}_{1}\right.$ y $\left.\mathrm{K}_{2}\right)$. La energía cinética del bloque del robot está compuesta por la energía cinética de la rotación (asociada a la velocidad angular del centro de masa) más la energía cinética de translación del cuerpo (asociado a la velocidad lineal del centro de masa) como se muestra en la ecuación 18, en la que el parámetro $\mathrm{I}_{3}=M\left(\mathrm{a}^{2}+\mathrm{b}^{2}+\mathrm{c}^{2}\right) / 12$, representa el momento de inercia del cuerpo del robot con respecto a su centro de giro.

$\mathrm{K}_{\mathrm{C}}=\frac{1}{2} \mathrm{I}_{3} \dot{\sim}^{2}+\frac{1}{2} \mathrm{MV}_{\mathrm{CM}}^{2}$

La energía cinética de cada rueda está asociada a un componente de rotación y otro de traslación como se escribe en la ecuación 19. 
$\mathrm{K}_{\mathrm{i}}=\frac{1}{2} \mathrm{l}_{\mathrm{i}} \dot{\theta}_{\mathrm{i}}^{2}+\frac{1}{2} \mathrm{mV}_{\mathrm{cm}}^{2}$

En la ecuación anterior el subíndice i representa el número de rueda que se está analizando (1 rueda derecha, 2 rueda izquierda) e $\mathrm{I}_{\mathrm{i}}=\mathrm{mr}^{2} / 2$ es el momento de inercia de cada rueda y $\mathrm{V}_{\mathrm{cm}}=\dot{\theta}_{\mathrm{i}} \mathrm{r}$ es la velocidad lineal del centro de masa de cada una de las ruedas como se muestra en la figura 5.

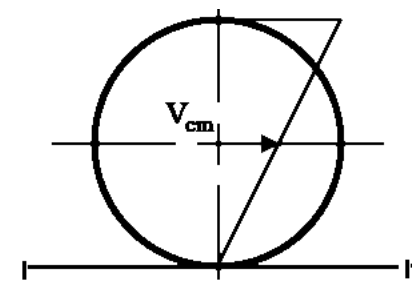

Fig. 5: Velocidad del centro de masa de la rueda del robot.

El Lagrangiano de las llantas del robot está compuesto por la suma de las energías cinéticas de rotación y traslación de las mismas, definido mediante la ecuación 20.

$\ell_{\mathrm{i}}=\frac{1}{2} \mathrm{I}_{\mathrm{i}} \dot{\theta}_{\mathrm{i}}^{2}+\frac{1}{2} \mathrm{mV}_{\mathrm{cm}}^{2}$

Reemplazando el resultado la velocidad del centro de masa $\mathrm{V}_{\mathrm{cm}}$ de cada rueda en la ecuación 21 se obtienen los Lagrangianos para cada rueda y se muestran en la ecuación 21.

$\ell_{\mathrm{i}}=\frac{1}{2} \mathrm{l}_{\mathrm{i}} \dot{\theta}_{\mathrm{i}}^{2}+\frac{1}{2} \mathrm{~m} \dot{\theta}_{\mathrm{i}}^{2} \mathrm{r}^{2}$

El Lagrangiano del cuerpo del robot está compuesto por la energía cinética de rotación del centro de masa y la de traslación del mismo tal como se muestra en la ecuación 22.

$\ell_{3}=\frac{1}{2} \mathrm{I}_{3} \dot{\psi}^{2}+\frac{1}{2} \mathrm{MV}_{\mathrm{CM}}^{2}$

Haciendo los reemplazos correspondientes se obtiene el Lagrangiano del cuerpo del robot en términos de las velocidades angulares de las ruedas según se muestra en la ecuación 23.

$\ell_{3}=M r^{2}\left(\frac{a^{2}+13 b^{2}+c^{2}}{96 b^{2}}\right)\left[\dot{\theta}_{1}^{2}+\dot{\theta}_{2}^{2}\right]-\operatorname{Mr}^{2}\left(\frac{1+12 b^{2}}{24}\right) \dot{\theta}_{1} \dot{\theta}_{2}$

Sumando las ecuaciones 21, 22 y 23 se obtiene el Lagrangiano total del robot mediante la ecuación 24.

$\ell=\left[\frac{3}{4} m r^{2}+\frac{M r^{2}\left(a^{2}+13 b^{2}+c^{2}\right)}{96 b^{2}}\right]\left(\dot{\theta}_{1}^{2}+\dot{\theta}_{2}^{2}\right)-M r^{2}\left(\frac{1+12 b^{2}}{24}\right) \dot{\theta}_{1} \dot{\theta}_{2}$

A continuación, se calculan los torques de acuerdo con el Modelado del robot, para ello se utiliza la coordenada generalizada $\theta_{1}$

$\frac{\mathrm{d}}{\mathrm{dt}} \frac{\partial \ell}{\partial \dot{\theta}_{1}}-\frac{\partial \ell}{\partial \theta_{1}}=\tau_{1}$

Calculando cada uno de los términos de esta ecuación se obtienen los siguientes resultados:

$\frac{\partial \ell}{\partial \dot{\theta}_{1}}=\dot{\theta}_{1}\left[\frac{3 m r^{2}}{2}+\frac{M^{2}\left(a^{2}+13 b^{2}+c^{2}\right)}{48 b^{2}}\right]-M r^{2}\left(\frac{1+12 b^{2}}{24}\right) \dot{\theta}_{1} \dot{\theta}_{2}$ 


$$
\begin{aligned}
& \frac{\mathrm{d}}{\mathrm{dt}}\left(\frac{\partial \ell}{\partial \dot{\theta}_{1}}\right)=\ddot{\theta_{1}}\left[\frac{3 m r^{2}}{2}+\frac{M r^{2}\left(\mathrm{a}^{2}+13 \mathrm{~b}^{2}+\mathrm{c}^{2}\right.}{48 \mathrm{~b}^{2}}\right]-\mathrm{Mr}^{2}\left(\frac{1+12 \mathrm{~b}^{2}}{24}\right) \ddot{\theta}_{2} \\
& \frac{\partial \ell}{\partial \theta_{1}}=0
\end{aligned}
$$

Utilizando la ecuación 25 se obtiene el torque que actúa en cada rueda, para la rueda uno la ecuación 29 y para la rueda dos la ecuación 31.

$$
\begin{aligned}
& \tau_{1}=\ddot{\theta}_{1}\left[\frac{3 m r^{2}}{2}+\frac{M r^{2}\left(a^{2}+13 b^{2}+c^{2}\right)}{48 b^{2}}\right]-M r^{2}\left(\frac{1+12 b^{2}}{24}\right) \ddot{\theta}_{2} \\
& \tau_{2}=\ddot{\theta}_{2}\left[\frac{3 m r^{2}}{2}+\frac{M r^{2}\left(a^{2}+13 b^{2}+c^{2}\right)}{48 b^{2}}\right]-M r^{2}\left(\frac{1+12 b^{2}}{24}\right) \ddot{\theta}_{1}
\end{aligned}
$$

El sistema anterior se puede expresar en forma matricial de la siguiente manera:

$$
\left[\begin{array}{l}
\tau_{1} \\
\tau_{2}
\end{array}\right]=\left[\begin{array}{ll}
K_{1} & K_{2} \\
K_{3} & K_{4}
\end{array}\right]\left[\begin{array}{l}
\ddot{\theta}_{1} \\
\ddot{\theta}_{2}
\end{array}\right]
$$

Donde:

$$
\mathrm{K}_{1}=\mathrm{K}_{4}=\left[\frac{3 m r^{2}}{2}+\frac{M r^{2}\left(\mathrm{a}^{2}+13 \mathrm{~b}^{2}+\mathrm{c}^{2}\right)}{48 \mathrm{~b}^{2}}\right], \mathrm{K}_{2}=\mathrm{K}_{3}=M r^{2}\left(\frac{1+12 \mathrm{~b}^{2}}{24}\right)
$$

Y así, las aceleraciones angulares se hallan utilizando la matriz inversa, mediante la ecuación 26.

$$
\left[\begin{array}{l}
\ddot{\theta}_{1} \\
\ddot{\theta}_{2}
\end{array}\right]=\left[\begin{array}{ll}
\mathrm{K}_{1} & \mathrm{~K}_{2} \\
\mathrm{~K}_{3} & \mathrm{~K}_{4}
\end{array}\right]^{-1}\left[\begin{array}{l}
\tau_{1} \\
\tau_{2}
\end{array}\right]
$$

Buscando que el modelo sea aún más exacto, se incluye el roce dinámico en cada rueda, modelado de forma simple como un par de torsión que ejerce una fuerza proporcional a la velocidad angular de cada una de las ruedas, la fuerza es contraria y la constante de proporcionalidad B, como se aprecia en la ecuación 34:

$$
\left[\begin{array}{l}
\ddot{\theta}_{1} \\
\ddot{\theta}_{2}
\end{array}\right]=\left[\begin{array}{l}
K_{1} K_{2} \\
K_{3} K_{4}
\end{array}\right]^{-1}\left[\begin{array}{l}
\tau_{1} \\
\tau_{2}
\end{array}\right]-\left[\begin{array}{l}
B \dot{\theta}_{1} \\
B \dot{\theta}_{2}
\end{array}\right]
$$

Integrando en el tiempo esta ecuación se pueden obtener las velocidades angulares de cada rueda, que tienen en cuenta las masas e inercias del robot necesarias para resolver las ecuaciones cinemáticas.

\section{MODELO ELECTROMECÁNICO}

El servomotor del robot LEGO NXT 2.0, es un tipo de motor con características especiales de control de posición, con componentes electromecánicos y electrónicos, que consta de un motor DC en su interior, y su eje está acoplado a un sistema de engranajes rectos que busca potenciar el torque del motor y permitir una posición fija cuando se requiera.

El modelo del motor de corriente directa (DC) se basa en las leyes de Kirchoff que permiten establecer la relación entre corriente consumida y torque entregado por los servomotores, así como la relación entre tensión y velocidad. En la ecuación $35, \mathrm{~V}$ representa la voltaje aplicada al motor, $\mathrm{R}$ es la resistencia interna, $\mathrm{L}$ su inductancia y e es la fuerza electromotriz inducida, i corresponde con la corriente que circula por el motor. 


$$
V=L \frac{d i}{d t}+R i+e
$$

En la ecuación 36 se escribe el torque aplicado $\tau_{m}$ al motor de DC que es proporcional a la corriente i(t) del circuito, $\mathrm{K}_{\mathrm{t}}$ es la constante de torque del motor, e corresponde a la tensión inducida que es proporcional a la velocidad angular del eje del motor $\mathrm{Wm}_{\mathrm{m}} \mathrm{y} \mathrm{K}_{\mathrm{E}}$ es la constante de armadura.

$\tau_{\mathrm{m}}=\mathrm{K}_{\mathrm{t}} \mathrm{i}(\mathrm{t}), \mathrm{e}=\mathrm{K}_{\mathrm{E}} \mathrm{W}_{\mathrm{m}}$

El acople de los motores a las ruedas del robot LEGO se realiza a través de una reducción por tren de engranes rectos, el torque en las ruedas $\tau$ es $G$ veces el torque en del motor, donde $G$ es la relación de engranes, mientras la velocidad angular de las ruedas se ve reducida $G$ veces con respecto a la velocidad del eje del motor, las revoluciones de los mismos se disminuyen, obteniendo de esta forma las ecuaciones mostradas en 37.

$$
\tau=\mathrm{G}_{\mathrm{m}}, \dot{\theta}=\frac{\mathrm{w}_{\mathrm{m}}}{\mathrm{G}}
$$

Al sustituir las ecuaciones 36 y 37 en la ecuación 35, se obtienen las ecuaciones electromecánica 38 y 39

$$
\begin{aligned}
& \mathrm{V}=\mathrm{L} \frac{\mathrm{di}}{\mathrm{dt}}+\mathrm{Ri}+\mathrm{K}_{\mathrm{E}} \mathrm{G} \dot{\theta} \\
& \tau=\mathrm{GK}_{\mathrm{t}} \mathrm{i}
\end{aligned}
$$

Utilizando las ecuaciones cinemáticas, dinámicas y electromecánicas obtenidas en las secciones anteriores se tiene un modelo matemático que describe el comportamiento del robot con base en la tensión y la corriente aplicados a cada motor, así las ecuaciones que describen el sistema van de la ecuación 40 a 47.

$$
\begin{aligned}
& \mathrm{L} \frac{\mathrm{di}}{\mathrm{dt}}=\mathrm{V}_{1}-\mathrm{Ri}_{1}-\mathrm{k}_{\mathrm{e}} \mathrm{G} \dot{\dot{\theta}_{1}} \\
& \mathrm{~L} \frac{\mathrm{di}}{\mathrm{dt}}=\mathrm{V}_{2}-\mathrm{Ri}_{2}-\mathrm{k}_{\mathrm{e}} \mathrm{G} \dot{\theta}_{2} \\
& {\left[\begin{array}{l}
\ddot{\theta}_{1} \\
\ddot{\theta}_{2}
\end{array}\right]=\left[\begin{array}{l}
\mathrm{K}_{1} \mathrm{~K}_{2} \\
\mathrm{~K}_{3} \mathrm{~K}_{4}
\end{array}\right]^{-1}\left[\begin{array}{l}
\mathrm{K}_{\mathrm{t}} \mathrm{Gi}_{1} \\
\mathrm{~K}_{\mathrm{t}} \mathrm{Gi}_{2}
\end{array}\right]-\left[\begin{array}{c}
\mathrm{B} \dot{\theta}_{1} \\
\mathrm{~B} \dot{\theta}_{2}
\end{array}\right], \mathrm{K}_{1}=\mathrm{K}_{4}=\frac{3 \mathrm{mr}^{2}}{2}+\frac{\mathrm{Mr}^{2}\left(\mathrm{a}^{2}+13 \mathrm{~b}^{2}+\mathrm{c}^{2}\right)}{48 \mathrm{~b}^{2}} \quad \mathrm{~K}_{2}=\mathrm{K}_{3}=\mathrm{Mr}^{2} \frac{1+12 \mathrm{~b}^{2}}{24}} \\
& \dot{\theta}_{1}(\mathrm{t})=\dot{\theta}_{1}(0)+\oint_{0}^{\mathrm{t}} \hat{\oint}_{1} \mathrm{dt} \\
& \dot{\theta}_{2}(\mathrm{t})=\dot{\theta}_{2}(0)+\dot{\oint}_{0}^{\mathrm{t}} \mathrm{dt} \\
& x(t)=x(0)+\int_{0}^{t} \frac{r\left(\dot{\theta}_{1}+\dot{\theta}_{2}\right)}{2} \operatorname{Cos}(\psi(t)) d t \\
& y(t)=y(0)+\int_{0}^{t} \frac{r\left(\dot{\theta}_{1}+\dot{\theta}_{2}\right)}{2} \operatorname{Sen}(\psi(t)) d t \\
& \psi(\mathrm{t})=\psi(0)+\int_{0}^{\mathrm{t}} \frac{\left.\dot{\theta}_{1}-\dot{\theta}_{2}\right)}{2 \mathrm{~b}} \mathrm{dt}
\end{aligned}
$$




\section{RESULTADOS}

Una forma de realizar la simulación es mediante el establecimiento de un conjunto de ecuaciones que puedan evaluarse para obtener el estado exacto del robot (Gómez, 2017), en este caso la ecuaciones de posición y orientación en función del tiempo. La simulación se realizó en Simulink de Matlab, y el diagrama de bloques se muestra en la figura 6, corresponde al modelo total del robot móvil LEGO, considerando los modelos cinemático, dinámico y electromecánico deducidos anteriormente. La posición inicial del centro de masa del robot para dicha simulación es: $\mathrm{X}=0, \mathrm{Y}=0, \psi=\pi / 4$. En cada simulación se muestran las tensiones de entrada aplicadas a cada servomotor, la posición final del centro de masa del robot con respecto al sistema XY y la orientación final del centro de masa del robot en el tiempo. En el diagrama de bloques es posible variar la orientación y la posición inicial del mismo. El tiempo considerado en la simulación es de 30 segundos.

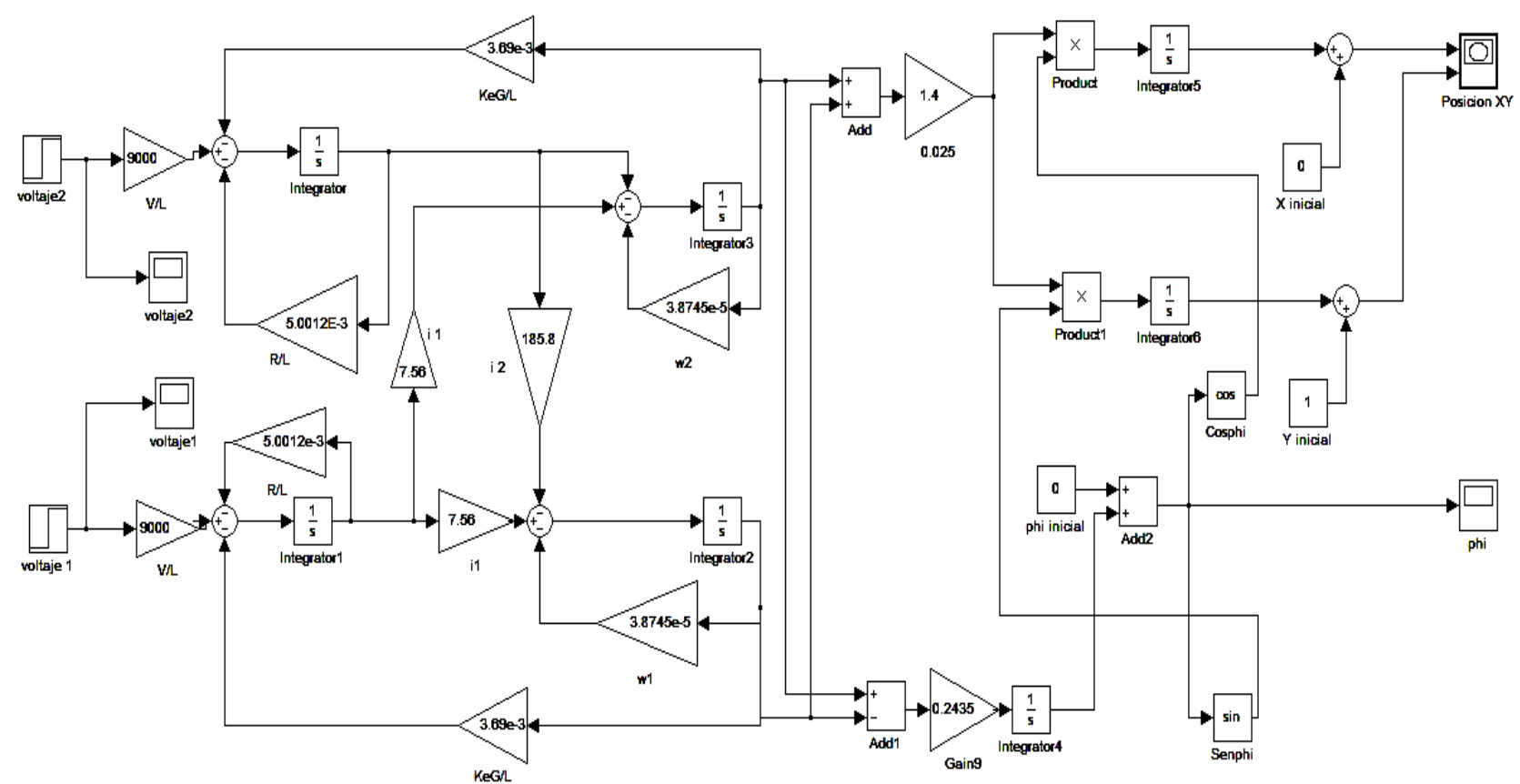

Fig.6: Diagrama de bloques en Simulink

Los parámetros eléctricos y geométricos del motor del robot LEGO, se presentan en la tabla 1 (Salamanca y Bermúdez, 2007).

Tabla 1: Parámetros geométricos, mecánicos y eléctricos del robot LEGO

\begin{tabular}{|l|l|l|l|}
\hline \multicolumn{1}{|c|}{ Parámetro } & \multicolumn{1}{|c|}{ Valores } & \multicolumn{1}{|c|}{ Parámetro } & \multicolumn{1}{|c|}{ Valores } \\
\hline $\begin{array}{l}\text { M=Masa del cuerpo del } \\
\text { robot. }\end{array}$ & $2,2 \mathrm{Kg}$ & $\begin{array}{l}\mathrm{K}_{\mathrm{e}}=\text { Constante fuerza } \\
\text { contraelectromotriz del motor }\end{array}$ & $0,246 \mathrm{~V} / \mathrm{rad} / \mathrm{s}$ \\
\hline $\begin{array}{l}2 \mathrm{~b}=\text { Distancia entre las } \\
\text { ruedas del robot. }\end{array}$ & $18 \times 10^{-2} \mathrm{~m}$ & $\begin{array}{l}\mathrm{N}=\text { Relación de la reducción de } \\
\text { velocidad de los motores }\end{array}$ & 15 \\
\hline $\begin{array}{l}\text { r=radio de las ruedas del } \\
\text { robot. }\end{array}$ & $2 \times 10^{-2} \mathrm{~m}$ & $\begin{array}{l}\text { J=Momento de Inercia del rotor del } \\
\text { motor }\end{array}$ & $2,4589 \mathrm{e}-6 \mathrm{Km}^{2}$ \\
\hline $\begin{array}{l}\mathrm{R}=\text { Resistencia óhmica de } \\
\text { los embobinados del } \\
\text { motor. }\end{array}$ & $5,0012 \Omega$ & $\begin{array}{l}\mathrm{I}_{\mathrm{z}}=\text { Momento de inercia del robot } \\
\text { alrededor del eje vertical } \mathrm{Z}\end{array}$ & $0,02197 \mathrm{Kg} \mathrm{\textrm {m } ^ { 2 }}$ \\
\hline $\begin{array}{l}\mathrm{L}=\text { Inductancia de los } \\
\text { embobinados del motor. }\end{array}$ & $10^{-3} \mathrm{H}$ & $\begin{array}{l}\text { B=Coeficiente de fricción viscosa } \\
\text { entre el rotor y el estator }\end{array}$ & $3,8745 \mathrm{e}-5 \mathrm{Nm} / \mathrm{rad} / \mathrm{s}$ \\
\hline $\begin{array}{l}\mathrm{K}_{\mathrm{t}}=\text { Constante de torque } \\
\text { del motor. }\end{array}$ & $0,246 \mathrm{Nm} / \mathrm{A}$ & m=Masa de las ruedas & $0,029 \mathrm{Kg}$ \\
\hline $\begin{array}{l}\text { a=Alto del bloque del } \\
\text { robot }\end{array}$ & $11 \times 10^{-2} \mathrm{~m}$ & c=Ancho del bloque del robot & $7 \times 10^{-2} \mathrm{~m}$ \\
\hline $\begin{array}{l}\text { b=Profundidad del bloque } \\
\text { del robot }\end{array}$ & $4 \times 10^{-2} \mathrm{~m}$ & & \\
\hline
\end{tabular}


Simulación 1: Las tensiones aplicadas a los motores son iguales $V_{1}=V_{2}=2,5 \mathrm{~V}$ como se muestra en la figura 7 , por tal razón el robot mantiene la misma velocidad angular en las ruedas. Se puede apreciar que el centro de masa del robot se mueve en una línea recta con inclinación de $\psi=\pi / 4$ en el plano XY, y el ángulo de posicionamiento se mantiene constante.
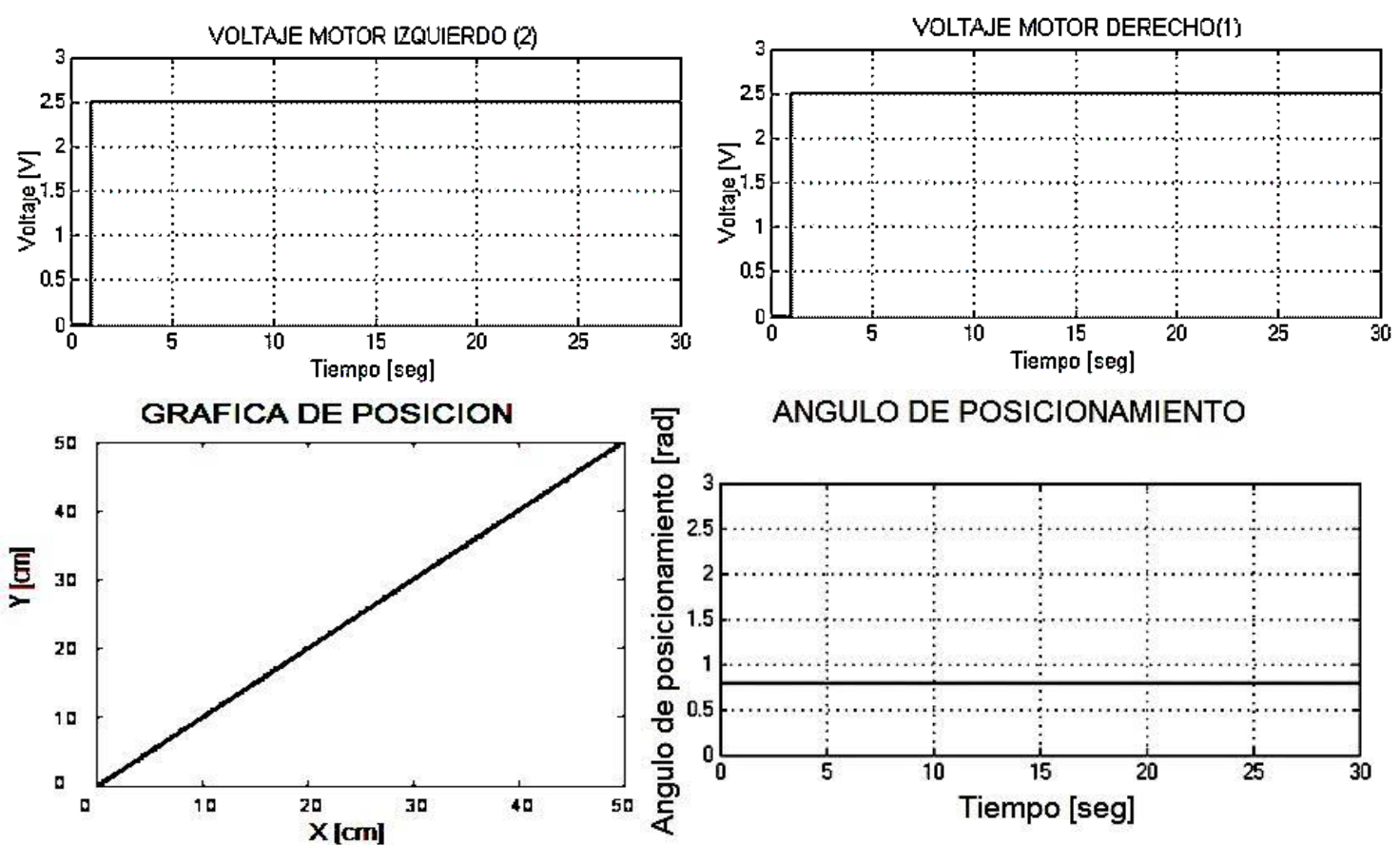

Fig. 7: Simulación para señales de voltaje iguales para cada motor

Simulación 2. En ésta simulación las tensiones aplicadas a los servomotores son diferentes, la tensión en el servomotor derecho es igual a 3,5 $\mathrm{V}$ y en el izquierdo es de $2,5 \mathrm{~V}$, debido a ello el centro de masa del robot móvil describe una trayectoria curva en sentido antihorario debido a que se desarrolla una velocidad angular mayor en la rueda derecha con respecto a la izquierda y el ángulo de posicionamiento aumenta a partir de su posición inicial (pi/4=0,7854) tal como se muestra en la figura 8 .
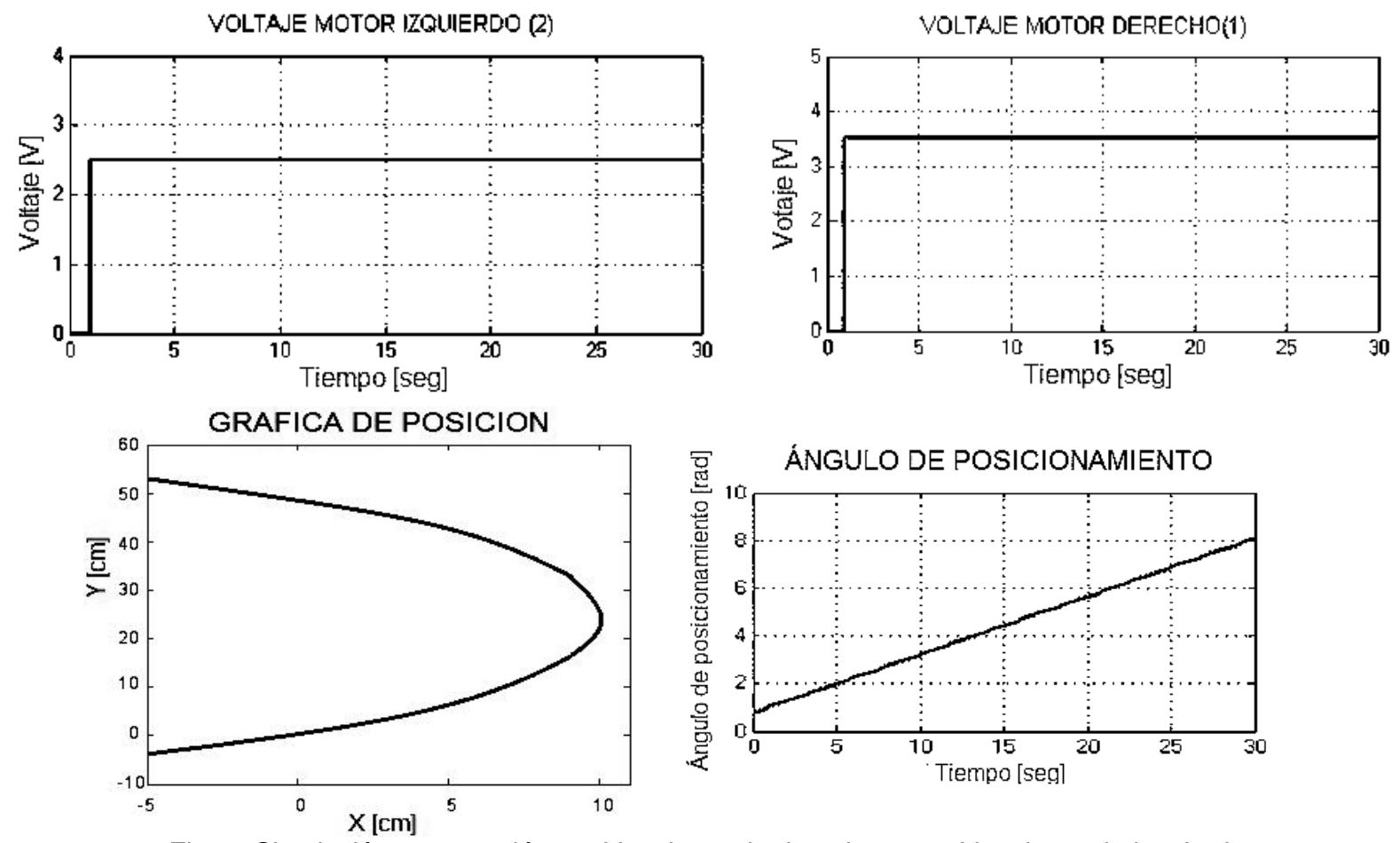

Fig. 8: Simulación para tensión 3,5 V en la rueda derecha, y 2,5 V en la rueda izquierda.

Simulación 3. En ésta simulación las tensiones aplicadas a los servomotores son diferentes, la tensión en el servomotor derecho es igual a $2,5 \mathrm{v}$ y en el izquierdo es de 3,5 v, debido a ello el centro de masa del robot 
móvil describe una trayectoria en sentido horario desde su posición de partida debido a que se desarrolla una velocidad angular mayor en el motor izquierdo con respecto al derecho y el ángulo de posicionamiento disminuye a partir de su posición inicial $(\psi=\pi / 4=0,7854)$ tal como se muestra en la figura 9.
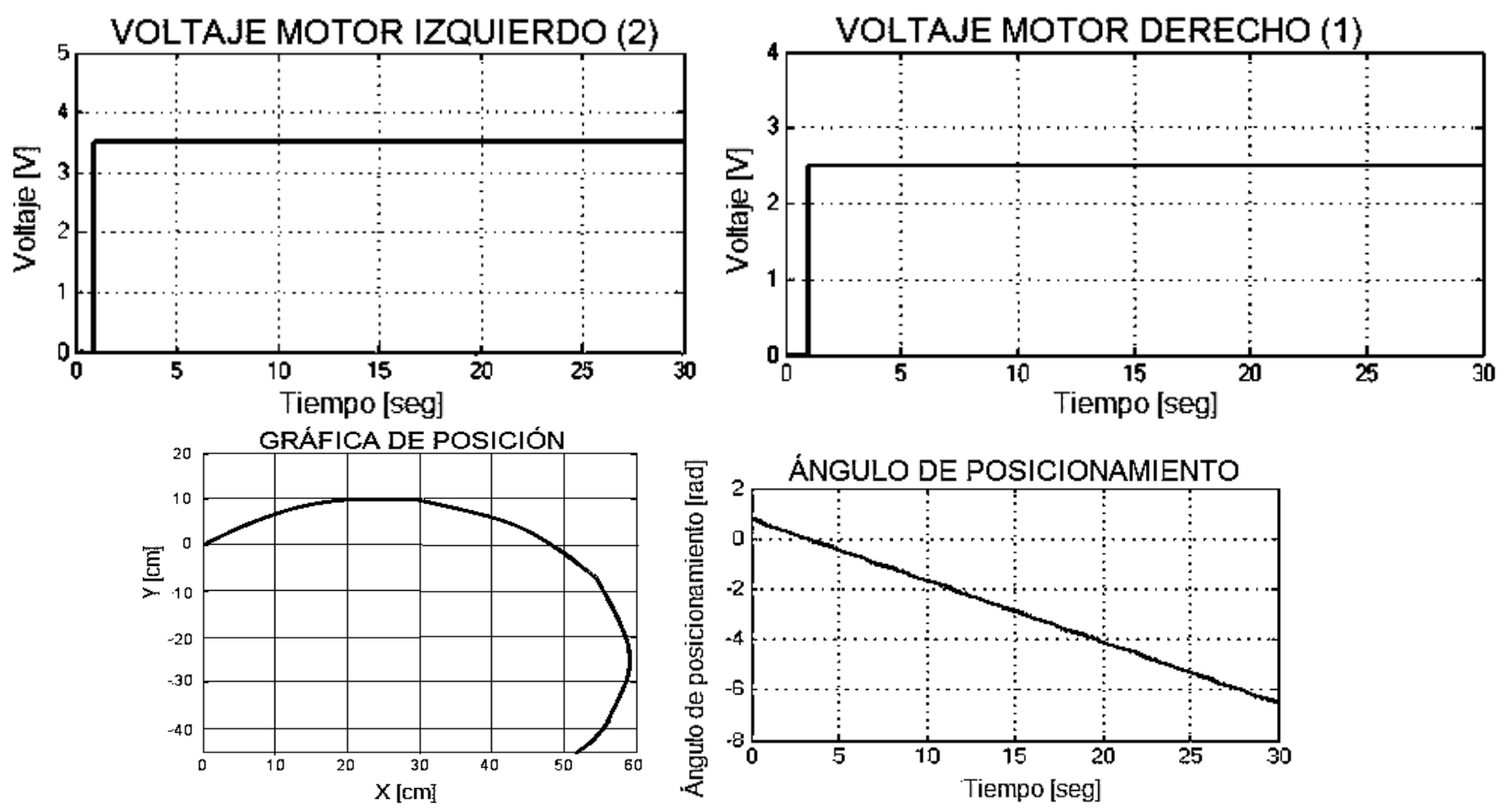

Fig. 9: Simulación para motor izquierdo con voltaje $3.5 \mathrm{~V}$ y derecho $2.5 \mathrm{~V}$

Simulación 4. En ésta simulación las tensiones aplicadas a los servomotores son iguales pero con polaridades opuestas, el voltaje en el servomotor derecho es igual a $2,5 \mathrm{v}$ y en el izquierdo es de $-2,5 \mathrm{v}$, debido a ello el centro de masa del robot móvil gira continuamente sobre un mismo punto y no se desplaza, sin embargo su ángulo de posicionamiento aumenta lo que significa que el robot se encuentra girando sobre su eje vertical dicha situación se muestra en la figura 10.
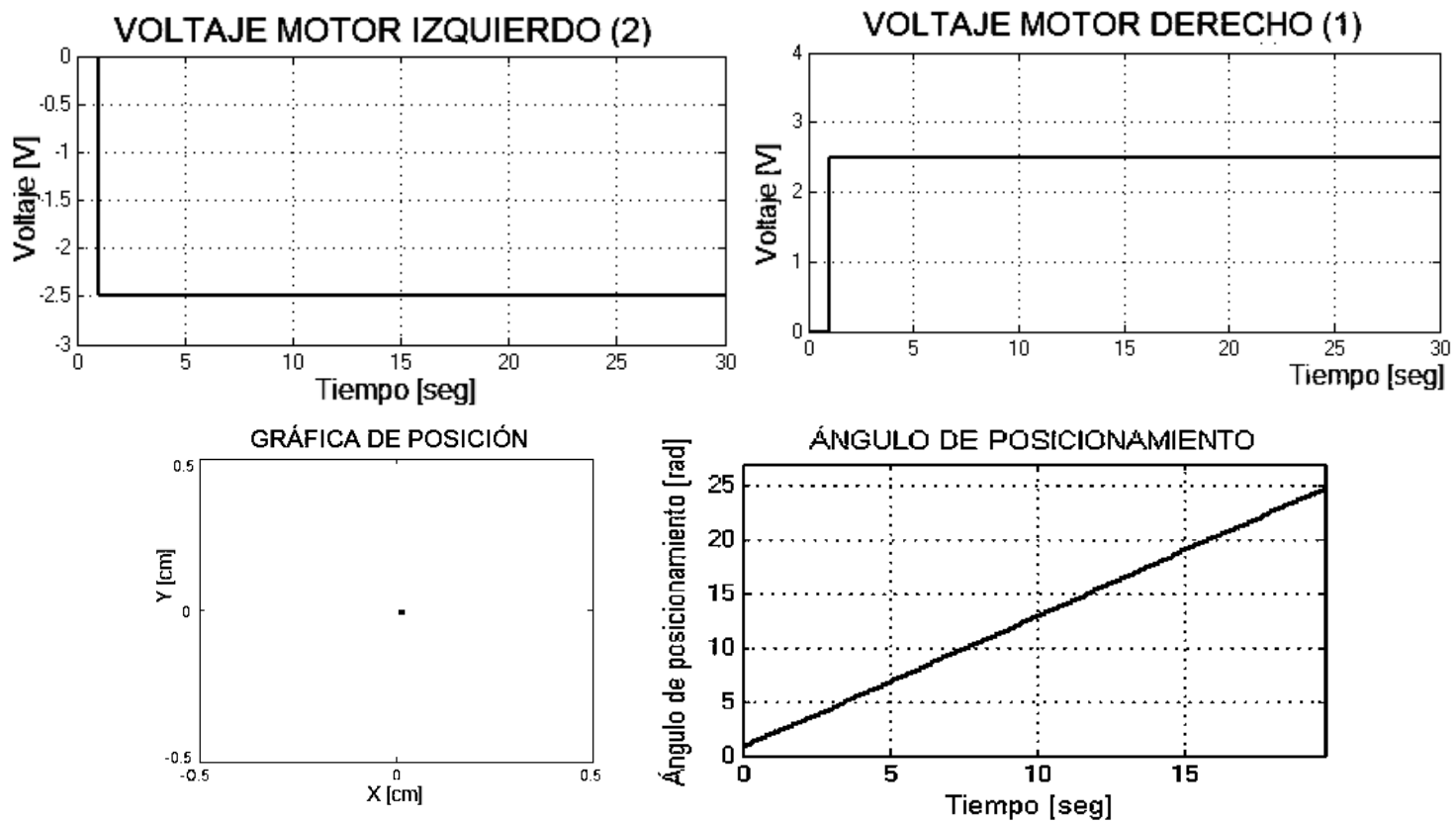

Fig. 10: Simulación para motor derecho con voltaje $2.5 \mathrm{~V}$ e izquierdo $-2.5 \mathrm{~V}$

\section{VALIDACIÓN DEL MODELO}

Se determinó experimentalmente el centro de masa del robot móvil diferencial, en primer lugar se sujeta el robot de alguna parte y se traza la proyección, luego se repite el procedimiento sujetando al robot desde otra parte, el punto de corte de las dos proyecciones trazadas sobre el robot determina el centro de masa del mismo, punto que luego se marca en color rojo. Una vez marcado el centro de masa, se utilizó el software Tracker para conocer los datos experimentales de trayectoria y orientación, el cual analiza el movimiento del 
punto marcado utilizando un video que lo rastrea cuando se mueve en una superficie plana. En la figura 11 se aprecia una captura del video analizado por el software.

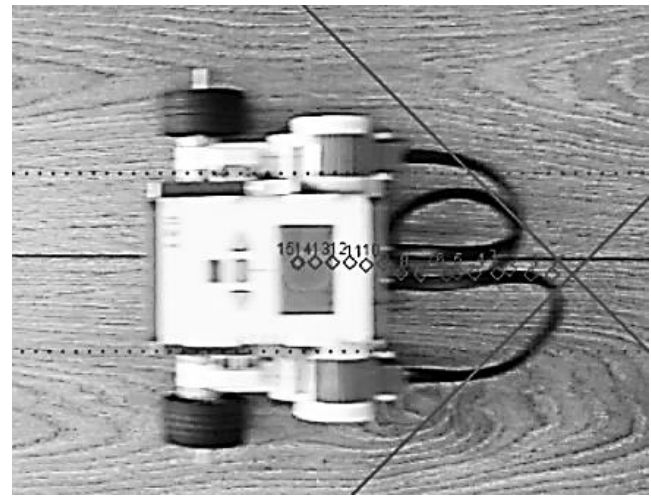

Fig. 11: Fotografía del robot en movimiento para la validación con el software Tracker

El experimento se realiza para la primera condición establecida en la simulación número uno, es decir cuando los motores se mueven a la misma velocidad angular recibiendo cada motor una tensión de 2,5 V. En el software se configura el sistema cartesiano a 45 grados con respecto a la trayectoria del centro de masa del robot con el objetivo de que el experimento se realice en la misma condición de simulación. Con el software es posible obtener los datos experimentales de posición y orientación del centro de masa de forma analítica y gráfica.

En la tabla 2 se muestran algunos datos obtenidos experimentalmente para la posición X, Y del centro de masa del robot que aproximan las posiciones reales " $x$ ", y " $y$ " de la simulación, no son exactamente iguales debido a factores que afectan el experimento como el rozamiento entre las ruedas y la superficie de desplazamiento, entre otras. La figura 12 muestra la posición del centro de masa del robot con los datos experimentales.

Tabla 2:Algunos resultados experimentales de posición del centro de masa del robot LEGO

\begin{tabular}{|l|l|l|l|l|l|l|l|l|l|l|l|l|}
\hline Posición $X[\mathrm{~cm}]$ & 0 & 1,13 & 2,38 & 3,88 & 4,39 & 5,62 & 6,64 & 7,22 & 8,66 & 9,86 & 11,42 & 12,31 \\
\hline Posición $Y[\mathrm{~cm}]$ & 0 & 1,16 & 2,39 & 3,33 & 4,25 & 5,50 & 6,54 & 7,15 & 8,72 & 9,77 & 10,16 & 11,27 \\
\hline Posición $X[\mathrm{~cm}]$ & 35,86 & 36,16 & 36,94 & 37,79 & 38,77 & 39,71 & 40,71 & 41,76 & & & & \\
\hline Posición $Y[\mathrm{~cm}]$ & 34,36 & 35,92 & 36,95 & 37,84 & 38,87 & 39,83 & 40,79 & 41,73 & & & & \\
\hline
\end{tabular}

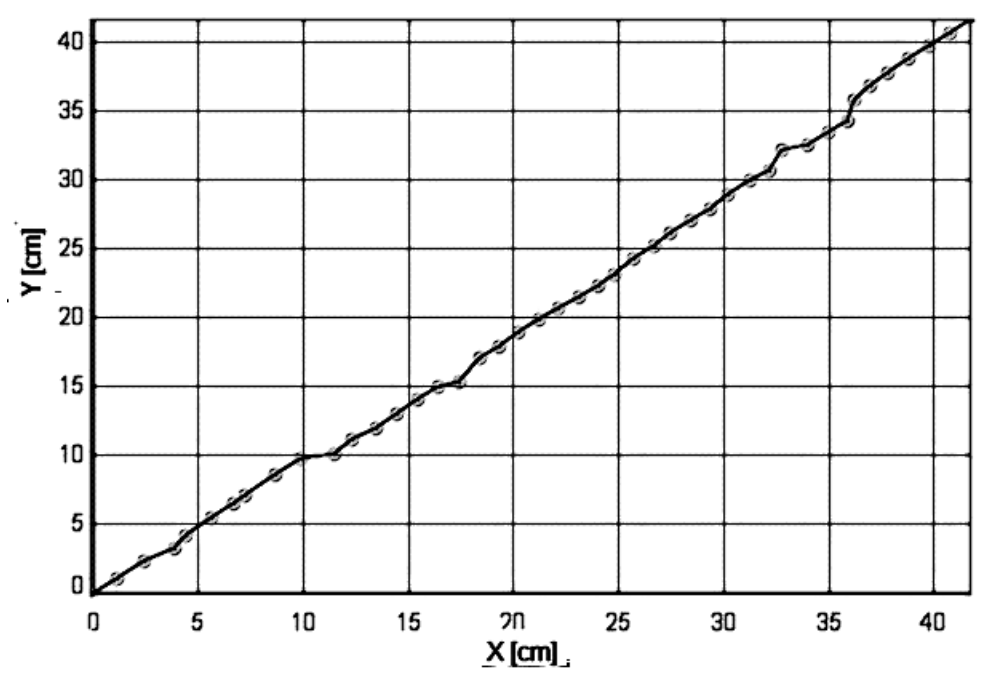

Fig. 12: Posición experimental del centro de masa del robot.

Para validar los datos obtenidos mediante la simulación del modelo matemático de la posición del centro de masa del robot se observa que con base en los resultados de la figura 7, la posición del centro de masa del robot está definida por una línea recta inclinada $45^{\circ}(\psi=\pi / 4)$, cuya ecuación corresponde a $Y=X$, lo que significa que una posición en $X$ corresponde exactamente a la misma posición sobre el eje $Y$, esto se debe a que las tensiones aplicadas a los servomotores son iguales, es decir que el modelo matemático tiene un comportamiento lineal. 
Una vez hecho esto, se comparan estadísticamente las posiciones experimentales del centro de masa del robot presentadas en la Tabla 2, con los datos de las simulaciones mediante una prueba t con un nivel de significancia del 0,05 , para ello se establecen las hipótesis nula $\mathrm{H}_{0}$ y alternativa $\mathrm{H}_{\mathrm{a}}$, respectivamente: $\mathrm{H}_{0}=$ "No hay diferencia significativa entre las medias experimentales y de simulación de la posición $\mathrm{x}$ y y del centro de masa del robot"; $\mathrm{H}_{\mathrm{a}}=$ "Hay diferencia entre las medias experimentales y de simulación de la posición x, y del centro de masa del robot"; El valor del estadístico de prueba t para dos colas que se obtiene es igual a 0,74 que es mayor al nivel de significancia de 0,05 por lo tanto se acepta la hipótesis nula, es decir que no hay diferencia significativa entre los valores de posición $\mathrm{X}, \mathrm{Y}$ simulados y experimentales del centro de masa del robot.

En la tabla 3 se muestran algunos datos experimentales obtenidos para la orientación del centro de masa del robot. En la figura 13 se muestra la gráfica de la orientación experimental obtenida con el software Tracker del centro de masa del robot.

Tabla 3: Algunos datos experimentales de la orientación del centro de masa del robot obtenidos mediante el software Tracker

\begin{tabular}{|l|c|c|c|c|c|c|c|c|c|c|c|c|c|c|}
\hline $\begin{array}{l}\text { Orientación } \\
\text { CM [rad] }\end{array}$ & 0,80 & 0,79 & 0,71 & 0,77 & 0,78 & 0,78 & 0,79 & 0,78 & 0,73 & 0,74 & 0,73 & 0,74 & 0,74 & 0,74 \\
\hline $\begin{array}{l}\text { Orientación } \\
\text { CM [rad] }\end{array}$ & 0,74 & 0,73 & 0,75 & 0,75 & 0,75 & 0,76 & 0,75 & 0,75 & 0,75 & 0,75 & 0,76 & 0,76 & 0,76 & 0,76 \\
\hline
\end{tabular}

Para validar el modelo matemático se comparan estadísticamente las orientaciones experimentales del centro de masa del robot y los datos obtenidos en la simulación mediante una prueba t con un nivel de significancia del 0.05 , para ello se establecen las hipótesis nula $\mathrm{H}_{\circ}$ y alternativa $\mathrm{H}_{a}$, de la siguiente manera: $\mathrm{H}_{\circ}=$ "No hay diferencia significativa entre las medias experimentales y las de simulación de la orientación del centro de masa del robot"; $\mathrm{H}_{\mathrm{a}}=$ "Hay diferencia entre las medias experimentales y las de simulación de la orientación del centro de masa del robot"; El valor del estadístico de prueba t para dos colas es igual a 1,66 que es mayor al nivel de significancia de 0,05 por lo tanto se acepta la hipótesis nula, es decir que no hay diferencia significativa entre los valores de orientación del centro de masa del robot X, Y experimentales y simulados.

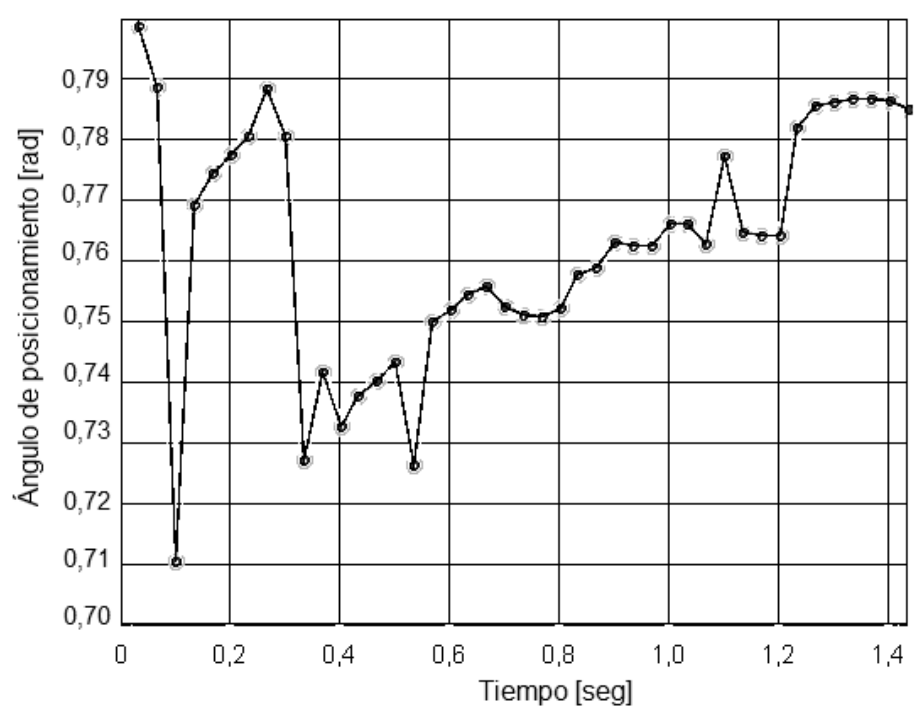

Fig.13: Orientación experimental del centro de masa del robot

\section{CONCLUSIONES}

A partir del trabajo presentado se puede concluir: 1) Al comparar los datos simulados con los resultados experimentales utilizando la prueba t-student, se encuentra que la diferencia porcentual entre los resultados experimentales y las simulaciones de posición y orientación del centro de masa es menor al 1\%. 2) El modelo diseñado y validado incluye variables de tipo cinemático, dinámico y electromecánico, con lo cual nos aproximamos a una simulación más cercana a la realidad. 3) Es posible determinar la posición y orientación del centro de masa del robot LEGO utilizando el software Tracker, pues la herramienta permite determinar tales variables y compararlas con los datos experimentales. 4) El modelo resulta ser más completo que los modelos cinemáticos estudiados ampliamente en la literatura especializada, pues incluye principios energéticos y variables inerciales del robot siendo aplicable a cualquier tipo de robot móvil diferencial, y sirve como base para el diseño del controlador. 


\section{REFERENCIAS}

Alexander, J. y J. Maddocks, On the Kinematics of Wheeled Mobile Robots, doi: 10.1177/027836498900800502, International Journal of Robotic Research - IJRR, 8, 15-27 (1989)

Buratowski, T. y J. Giergiel, Dynamics Modeling and Identification of the Amigobot Robot, Mechanics and Mechanical Engineering, ISSN: 2354-0192, 14(1), 65-79 (2010)

Campion, G., G. Bastin y B. Dandrea-Novel, Structural Properties and Classification of Kinematic and Dynamic Models of Wheeled Mobile Robots, doi: 10.1109/ROBOT.1993.292023, IEEE transactions on robotics and automation, 12(1), 47-62 (1996)

Chung, Y., C. Park y F. Harashima, A Position Control Differential Drive Wheeled Mobile Robot, doi: 10.1109/41.937419, IEEE Transactions on Industrial Electronics, 48(4), 853-863 (2001)

Esmaeili, N., A. Alfi y H. Khosravi, Balancing and Trajectory Tracking of Two-Wheeled Mobile Robot Using Backstepping Sliding Mode Control: Design and Experiments, doi: 10.1007/s10846-017-0486-9, Journal of Intelligent and Robotic Systems, 87(3-4), 1-13 (2017)

Fierro, R. y F.L. Lewis, Control of a Nonholonomic Mobile Robot: Backstepping Kinematics into Dynamics, Proceedings of the 34th IEEE Conference in Decision and Control 1995, 3805-3810, New Orleans, LA, USA, 13-15 Diciembre (1995)

Gómez, O.F. y U.E. Gómez, Simulación Cinemática de un Robot Seguidor de Línea para el Desarrollo del Videojuego de Programación Rusty Roads en el Framework Unity. doi: 10.4067/S0718-07642017000500008, Información Tecnológica, 28(5), 55-64 (2017)

Gomez-de-Gabriel, J.M., A. Mandow y otros dos autores, Using LEGO NXT Mobile Robots with LabVIEW for undergraduate courses on mechatronics, doi: 10.1109/TE.2010.2043359, IEEE Transactions on Education, 54(1), 41-47 (2011)

Granosik, G., M.G. Hansen y J. Borenstein, The Omnitread Serpentine Robot for Industrial Inspection and Surveillance. ISSN: 0143-991X, Industrial Robot: An International Journal, 32(2), 139-148 (2005)

Klančar, G., B. Zupančič y R. Karba, Modelling and Simulation of a Group of Mobile Robots, doi: 10.1016/j.simpat.2007.02.002, Simulation Modelling Practice and Theory, 15(6), 647-658 (2007)

Mahler, B. y J. Haase, Mathematical Model and Control Strategy of a Two-Wheeled Self-Balancing Robot, IECON 201339th Annual Conference of the IEEE In Industrial Electronics Society, 4198-4203, Vienna, Austria, 10-13 Noviembre (2013)

Muir, P. y C. Neuman, Kinematic Modeling for Feedback Control of an Omnidirectional Wheeled Mobile Robot, Proceedings of the 1987 IEEE International Conference on Robotics and Automation, 1772-1778, Raleigh, NC, USA, 1 de Marzo (1987)

Ortigoza, R.S., M. Marcelino-Aranda y otros cinco autores, Wheeled Mobile Robots: a review, doi: 10.1109/TLA.2012.6418124, IEEE Latin America Transactions, 10(6), 2209-2217 (2012)

Salamanca, M.L. y G.R. Bermúdez, Determinación de Parámetros de un Robot Móvil de LEGO Mindstorms ${ }^{\circledR}$, Ingeniería Investigación y Desarrollo, ISSN: 1900-771X, 5(2), 7-13 (2007)

Salinas, S.A. y A.O. Vivas, Modelado, Simulación y Control del Robot para Cirugía Laparoscópica Lapbot, doi: 10.4067/S0718-33052009000300005, Ingeniare, Revista Chilena de Ingeniería, 17 (3), 317-328 (2009)

Salem, F.A., Dynamic and kinematic Models and Control for Differential Drive Mobile Robots, International Journal of Current Engineering and Technology, ISSN: 2347-5161, 3(2), 253-263 (2013)

Todd, D.J., Walking Machines: an introduction to legged robots, $1^{\text {a }}$ Ed., Springer, USA (1985) 